\title{
Efficient Query Subscription Processing for Prospective Search Engines
}

\author{
Utku Irmak * Svilen Mihaylov ${ }^{\dagger} \quad$ Torsten Suel * \\ Samrat Ganguly $\ddagger \quad$ Rauf Izmailov $\ddagger$
}

\begin{abstract}
Current web search engines are retrospective in that they limit users to searches against already existing pages. Prospective search engines, on the other hand, allow users to upload queries that will be applied to newly discovered pages in the future. Some examples of prospective search are the subscription features in Google News and in RSS-based blog search engines.

In this paper, we study the problem of efficiently processing large numbers of keyword query subscriptions against a stream of newly discovered documents, and propose several query processing optimizations for prospective search. Our experimental evaluation shows that these techniques can improve the throughput of a well known algorithm by more than a factor of 20 , and allow matching hundreds or thousands of incoming documents per second against millions of subscription queries per node.
\end{abstract}

\section{Introduction}

The growth of the world-wide web to a size of billions of pages, and the emergence of large-scale search engines that allow real-time keyword searches over a large fraction of these pages, has fundamentally changed the manner in which we locate and access information. Such search engines work by downloading pages from the web and then building a full-text index on the pages. Thus, they are retrospective in nature, as they allow us only to search for currently already existing pages - including many outdated pages. In contrast, prospective search allows a user to upload a query that will then be evaluated by the search engine against documents encountered in the future. In essence, the user subscribes to the results of the query. The user can be notified of new matches in one of several ways, e.g., via email or an RSS reader.

A naive implementation of a prospective search engine

${ }^{*}$ CIS Department, Polytechnic University, Brooklyn, NY 11201. \{uirmak@cis.poly.edu, suel@poly.edu\}. The third author was also partially supported by NSF Awards IDM-0205647 and CCR-0093400, and the New York State Center for Advanced Technology in Telecommunications (CATT) at Polytechnic University.

${ }^{\dagger}$ CIS Department, University of Pennsylvania, Philadelphia, PA 19104. svilen@ seas. upenn.edu.

‡NEC Laboratories America, Inc., Princeton, NJ 08540. \{samrat@nec-labs.com, rauf@nec-labs.com\}. might simply execute all the subscription queries periodically against any newly arrived documents. However, if the number of subscriptions is very large, this would result either in a significant delay in identifying new matches, if we only execute the queries very rarely, or a significant query processing load for the engine. Following the approach in [11], we essentially reverse the roles of the documents and the queries. That is, we build an inverted index on the subscriptions instead of the documents, and then issue a number of queries into the index for each newly arriving document. We note, however, that the two cases are not completely symmetric. In this paper, we study techniques for optimizing the performance of prospective search engines. A more detailed version is available from the first author.

Applications of Prospective Search: One of the popular implementations of prospective search is the News Alert feature in Google News. It allows users to subscribe to a keyword search, in which case they will be notified via email of any newly discovered results matching all the terms. Similar services include specialized search applications created for job or real estate searches. Prospective search can be performed with the help of RSS (RSS 2.0: Really Simple Syndication) feeds, which allow web sites to syndicate their new content at a specified URL. Thus, a prospective search engine can find the new content on a site by periodically downloading the appropriate RSS feed. There are a number of existing weblog and RSS search engines based on RSS feeds, including PubSub, Bloglines, Technorati, and Feedster.

Problem Setup: We are given $n$ queries $q_{0}$ to $q_{n-1}$, where each query $q_{i}$ contains $s_{i}$ terms (keywords) $t_{i, 0}, \ldots, t_{i, s_{i}-1}$. We define $T$ as the union of all the $q_{i}$, i.e., the set of terms that occur in at least one query. The terms in a query may be arranged in some Boolean formula, though we will mainly focus on the AND queries. Given these queries, we are allowed to precompute appropriate data structures, say, an inverted index.

After preprocessing the queries, we are presented with a sequence of documents $d_{0}, d_{1}, \ldots$, where each document $d_{j}$ is a set of terms. We assume that $d_{j} \subseteq T$ for all $j$; this can be enforced by pruning from $d_{j}$ any terms that do not occur in any query $q_{i}$. We process the documents one by one where for each $d_{j}$ we have to determine all $q_{i}$ such that $d_{j}$ matches $q_{i}$. Within our matching system, 
queries are assigned integer query IDs (QID), and documents are assigned integer document IDs (DID), and all terms in the queries and documents are replaced by integer term IDs (TID). The output of the matching process is a stream of (QID, DID) pairs indicating matches.

While retrospective search engines typically store their index on disk, we assume that for prospective search, all the index structures fit into main memory. Tens of millions of queries can be kept in memory on a single machine with the currently available memory sizes. In case of more queries, our results indicate that CPU cycles become a bottleneck before main memory.

Discussion of Query Semantics: Most current search engines assume AND semantics, where a query matches any document containing all query terms, in combination with ranking. As we show, AND queries can be executed very efficiently in an optimized system for prospective search; moreover, several other interesting types of queries can be efficiently reduced to AND queries. Therefore, we focus on AND queries.

Boolean queries involve a number of terms connected by AND, OR, and NOT operators. In our framework, they are executed by converting them to DNF, inserting each conjunction as a separate AND query, and then removing duplicate matches from the output stream.

In the RSS search scenario, it might be preferable to restrict the keyword queries to certain fields. It is well understood that inverted index structures with appropriate extensions can efficiently process many such queries for retrospective search, and similar extensions are also possible for prospective search.

\section{Contributions of this Paper:}

- We describe the design of a high-performance subscription matching processor based on an inverted index, with several optimizations based on term frequencies and a Bloom filter structure.

- We study preprocessing schemes that cluster subscriptions into superqueries.

- We evaluate our schemes against a search engine trace, and detail the performance improvements created by our optimizations.

\section{The Core Query Processor}

In our query processor, TIDs are assigned from 0 to $|T|-1$, and the terms in each query are ordered by TID; thus we can refer to the first, second, etc., term in a query. Any incoming documents have already been preprocessed by parsing out all terms, translating them into TIDs, and discarding any duplicate terms or terms that do not occur in any query. It will also be convenient to assume that QIDs are assigned at random. Note that we expect additions and deletions of subscriptions to be handled in an amortized fashion, by periodic rebuilding of the structures (including updating the assignments of TIDs and QIDs).

The main data structure used in all our algorithms is an inverted index, which is also used by retrospective search engines. However, in our case we index the queries rather than the documents, as proposed in [11]. The inverted index consists of $|T|$ inverted lists, one for each unique term that occurs in the queries. Each list contains one posting for each query in which the corresponding word occurs, where a posting consists of the QID and the position of the term in the query (recall that terms are ordered within each query by TID). The QID and position can usually be stored together in a single 32-bit integer, and thus each inverted list is a simple integer array.

\subsection{A Primitive Matching Algorithm}

We now describe the primitive matching algorithm, which (with some variations) has been studied in a number of previous works including $[8,11,9,7]$. The basic idea is as follows. We initially build the inverted index from the queries using standard index construction algorithms; see, e.g., [10]. We also reserve space for a hash table, indexed by QIDs, of some sufficient size. Given an incoming document consisting of a set of terms, we first clear the hash table, and then process the terms in the document in some sequential order. To process a term, we traverse the corresponding inverted list in the index. For each posting of the form (QID, position) in this list, we check if there is already an entry in the hash table for this QID. If not, we insert such an entry into the hash table, with an associated accumulator (counter) set to 1 . If an entry already exists, we increase its accumulator by 1 . This first phase is called the matching phase.

In the second phase (testing phase), we iterate over all created hash table entries. For every entry, we test if the final value of the accumulator is equal to the number of query terms; if so then we output the match between this query and the document. Note that for Boolean queries other than AND, we could reserve one bit in the accumulator for each term in the query, and then instead of increasing a counter we set the corresponding bit; in the testing phase we check if the accumulator matches the query through tests applying appropriate bit masks. Also, since QIDs are assigned at random, we can use the QID itself as our hash function for efficiency.

\subsection{Optimizations for Primitive Algorithm}

Exploiting Position Information and Term Frequencies: One problem with the primitive algorithm is that it 
creates an entry in the hash table for any query that contains at least one of the terms. This results in a larger hash table that in turn slows down the algorithm, due to additional work that is performed but also due to resulting cache misses during hash lookups.

To decrease the size of the hash table, we first exploit the fact that we are focusing on AND queries. Recall that each index posting contains (QID, position) pairs. Thus, if we are processing a posting with a non-zero position, then this means that the term is not the term with the lowest TID in the query. Suppose we process the terms in the incoming document in sorted order, from lowest to highest TID. This means that for a posting with nonzero position, either there already exists a hash entry for the query, or the document does not contain any of the query terms with lower TID, and thus the query does not match. So we create a hash entry whenever the position in the posting is zero, and only update existing hash entries otherwise. As we will see, this results in significantly smaller hash table sizes. A further reduction is achieved by simply assigning TIDs to terms in order of frequency, that is, we assign TID 0 to the term that occurs the least frequent in the set of queries, and TID $|T|-1$ to the most frequent term. This means that an accumulator is only created for those queries where the incoming document contains the least frequent term in the query.

To implement this efficiently, we split each inverted list into two parts, a smaller list containing only postings with positions equal to zero, and the rest of the list. We then perform two passes over the terms in the incoming document, the first pass generates the hash entries, and the second pass updates the existing entries. This simplifies the critical inner loop over the postings and also allows us to quickly determine the optimal hash table size for each incoming document, by summing up the lengths of the first parts of the inverted lists involved.

Bloom Filters: As a result of the previous set of optimizations, hash entries are only created initially, and most of the time is spent afterwards on lookups to check for existing entries. Moreover, most of these checks are negative, i.e., the corresponding entry does not exist. In order to speed up these checks, we propose to use a Bloom filter $[2,3]$, which is a probabilistic spaceefficient method for testing set membership.

We use a Bloom filter in addition to the hash table. In the matching phase, when hash entries are created, we also set the corresponding bits in the Bloom filter; the overhead for this is fairly low. In the testing phase, we first perform a lookup into the Bloom filter to see if there might be a hash entry for the current QID. If the answer is negative, we immediately continue with the next posting; otherwise, we perform a lookup into the hash table.

Use of a Bloom filter has two advantages. The Bloom filter structure is small and thus gives better cache behavior, and the innermost loop of our matching algorithm is also further simplified. We experimented with different settings for the size of the Bloom filter and the number of hash functions; our results indicate that a single hash function (trivial Bloom filter) performs best.

Partitioning the Queries: We note that the hash table and Bloom filter sizes increase linearly with the number of query subscriptions, and thus eventually grow beyond the L1 or L2 cache sizes. This leads to our next optimization. Instead of creating a single index, we partition the queries into a number $p$ of subsets and build an index on each subset. In other words, we partition the index into $p$ smaller indexes. An incoming document is then processed by performing the matching sequentially with each of the index partitions. While this does not decrease the number of postings traversed, or the locality for index accesses, it means that the hash table and Bloom filter sizes that we need are decreased by a factor of $p$.

\subsection{Experimental Evaluation}

Since we were unable to find any large publicly available query subscription logs, we decided to use Excite search engine query logs, collected in 1999 . We note that query subscriptions in a prospective engine would likely be different in certain ways from standard retrospective queries; in particular, we would not expect as many extremely broad queries. For this reason, we will also look at how performance changes with query selectivity, by looking at different subsets of the query logs. To be used as incoming documents, we selected 10,000 web pages at random from a large crawl of over 120 million pages from Fall 2001.

We removed stop words and duplicate queries from the query trace, and also converted all the terms to lower case. We also removed queries that contained more than 32 terms; there were only 43 such queries out of a total of $1,077,958$ distinct queries. Some statistics on the query logs, documents, and resulting inverted index lookups is as follows: There are 271,167 unique terms in the query $\log$, and each query contains about 3.4 terms on average. The number of postings in the index is $3,633,970$. Each incoming document contains about 144 distinct terms that also occur in the queries. For each document, our algorithms will visit about 200,000 postings, or about 1,400 postings per inverted list that is traversed. Of those postings, only about 6,630 have position zero if we assign TIDs according to term frequencies.

To experiment with numbers of queries beyond the size of the query $\log$, we replicated the queries several times according to a multiplier between 1 and 14, for a maximum size of about 15 million queries. We note that 
the core query processor does not exploit similarities between different queries, and thus we believe that this scaling approach is justified. Later, in Section 3, we will incorporate clustering techniques into our system that exploit such similarities; for this reason we will not use a multiplier in the later evaluation of the clustering schemes, which limits us to smaller input sizes.

In the experiments, we report the running times of the various optimizations when matching different numbers of queries against 10, 000 incoming documents. The experiments are performed on a machine with a $3.0 \mathrm{Ghz}$ Pentium4 processor with $16 \mathrm{~KB} \mathrm{L1}$ and 2 MB L2 cache, under a Linux 2.6.12-Gentoo-r10 environment. We used the gcc compiler with Pentium4 optimizations. We also used the vtune performance tools to analyze program behavior such as cache hit ratio etc. In the charts, we separately show the times spent on the matching and testing phases. Note that the matching phase includes all inverted index traversals and the creation and maintenance of the accumulators, while the testing phase merely iterates over the created accumulators to identify matches.

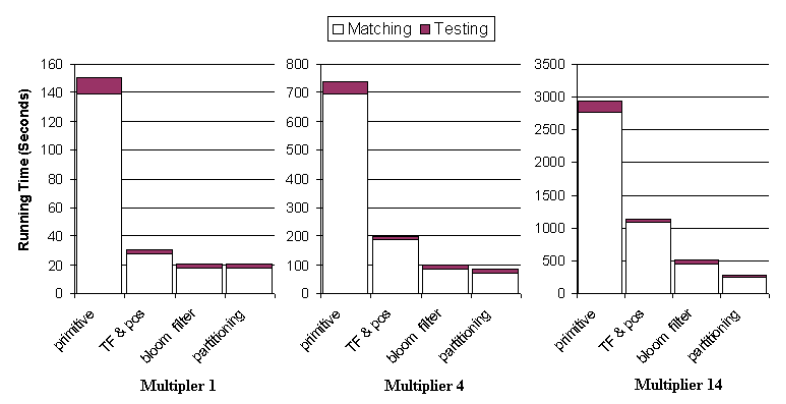

Figure 2.1: Running times of the various algorithm optimizations for different numbers of queries.

In Figure 2.1, we show the time spent by four versions of our query processor: (i) the primitive one, (ii) with optimization for AND and assignment of TIDs by frequency, (iii) with Bloom filter, and (iv) with index partitioning with optimal choice of the number of partitions. We show total running times in seconds for matching 10,000 documents against the queries with multipliers of 1, 4, and 14. At first glance, running times are roughly linear in the number of queries. More exactly, they are slightly more than linear for the first three algorithms, due to the increased sizes of the hash table and Bloom filter structures resulting in more cache misses, while the best algorithm (iv) remains linear by increasing the number of partitions.

As discussed, many Excite queries may have much larger result sizes than typical subscription queries would have. To examine possible relationships between matching performance and query selectivities, we partitioned our queries into quintiles according to selectivity. To do so, we matched all queries against a larger collection of around 144, 000 documents (disjoint from the set of 10,000 we used for testing), and counted the number of matches of each query. We then partitioned queries into five subsets, from the $20 \%$ with the fewest number of matches to the $20 \%$ with the most. In the Figure 2.2, we show how the running times of the algorithms change as we go from queries with very few results (leftmost 4 bars) to queries with very many results (rightmost 4 bars). Not surprisingly, queries with many matches are more costly. (Since we use a multiplier of 1 , the partitioning does not seem to give any benefits in the figure.)

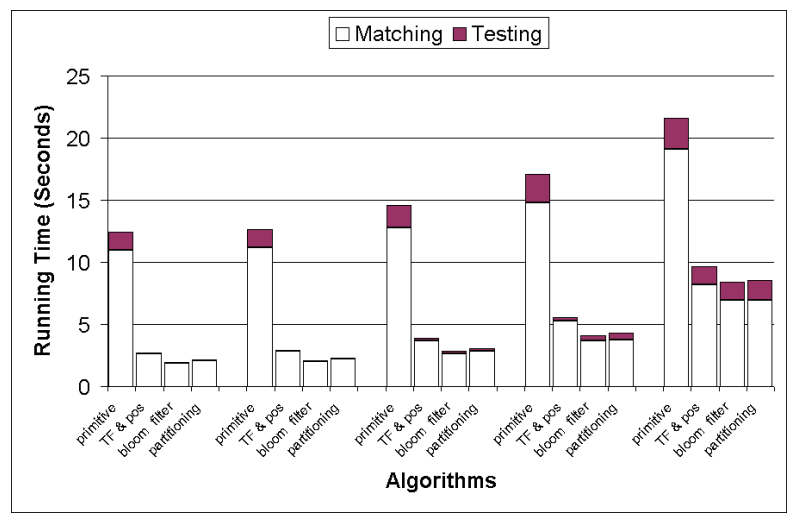

Figure 2.2: Running times versus query selectivities for the various algorithms, with multiplier 1.

To illustrate the benefits of index partitioning, we performed additional runs on a machine with $512 \mathrm{~KB}$ instead of $2 \mathrm{MB}$ of L2 cache. As shown in Figure 2.3, index partitioning resulted in a gain by about a factor of 4 for the largest query set. On the $2 \mathrm{MB}$ machine, a similar effect is expected for larger query multipliers.
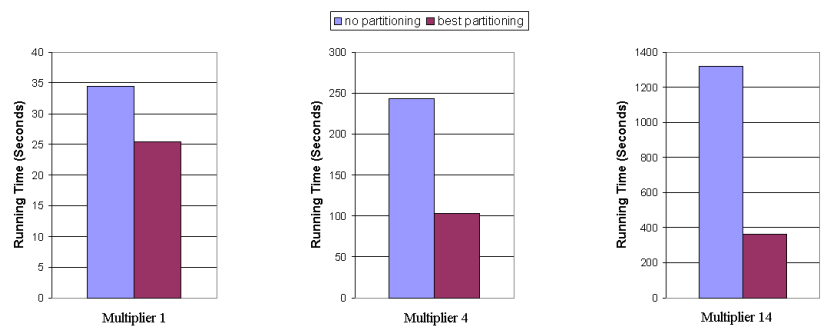

Figure 2.3: Benefit of best possible index partitioning on a machine with smaller L2 cache.

\section{Optimizations using Query Clustering}

In this section, we study clustering techniques to obtain additional performance benefits. We note that clustering of subscriptions has been previously studied, e.g., in [8], but in the context of more structured queries. Our simple approach for clustering subscriptions is as follows. In a preprocessing step, we cluster all queries into artificial superqueries of up to a certain size, such that every 
query is contained in a superquery and shares the same least frequent term with a superquery (and thus with all other queries in the cluster). Then we present these superqueries to the query processor, which indexes them and performs matching exactly as before. Thus the resulting improvements are orthogonal to the earlier techniques. The only changes to the core subscription processor are as follows: (i) During matching, we set appropriate bits in the accumulators for each update instead of counting, and (ii) in the testing phase we need to test each query that is contained in a superquery that has an accumulator. To do this test, we create a simple structure for each superquery during preprocessing that contains a list of the QIDs of the contained queries, plus for each QID a bit mask that can be used to test the superquery accumulator for this subquery.

We now discuss briefly how clustering impacts the cost of matching. Consider any two queries (or superqueries) that share the same least frequent term. Note that by combining these two queries into one superquery, we are guaranteed to decrease the size of the index by at least one posting. Moreover, the number of hash entries created and accumulator updates performed during the matching phase will never increase but may often decrease as a result of combining the two queries. On the other hand, we need at least one bit per term in the superquery for the accumulator, and a very large superquery would result in higher costs for both testing and hash table accesses. However, this effect is not easy to capture with a formal cost model for clustering. Instead, we will impose an upper bound $b$ on the size of each superquery, and try to minimize index size under this constraint. In general, this problem is still intractable, and we will focus on some heuristics.

\subsection{Greedy Algorithms for Clustering}

All the algorithms we present in this subsection follow the clustering approach discussed above. They start out by initially grouping all queries into pools based on their least frequent terms, and then separately build superqueries within each pool. Note that if we use index partitioning, we should make sure to assign all queries in a pool to the same partition.

Random Selection: The simplest approach starts with the empty superquery and then repeatedly picks an arbitrary query from the pool and merges it into the superquery, as long as the resulting superquery has at most $b=32$ terms (apart from the least frequent term). If the result has more than $b$ terms, then we write out the old superquery and start a new one.

Alphabetical Ordering: We first sort all queries in the pool in reverse alphabetical order - recall that the terms in each query are sorted by frequency and thus we sort according to most common, second most common, etc. terms. We then consider queries in sorted order as we construct superqueries, rather than in arbitrary order.

Overlap Ratio: The third greedy algorithm builds superqueries by checking all remaining queries in the pool to find the one with the best match, i.e., a query that has a lot of terms in common with the current superquery.

\subsection{Experimental Evaluation}

We cannot use the multiplier method of Section 2.3 in the evaluation of the proposed clustering algorithms since they exploit the similarities among the queries in the collection. In order to obtain at least a slightly larger collection of queries, we combined the Excite queries with another set of queries from the AltaVista search engine. The combined set contains 3,069, 916 queries and 922,699 unique terms. The number of postings in the resulting index is $9,239,690$. We first compare the performance of the proposed clustering algorithms in Table 3.1 when $b$ is set to 32 terms. The best algorithm from the previous section is shown on the last row. Even the most naive clustering algorithm gives significant benefits, and the algorithm based on overlap outperforms all others.

\begin{tabular}{|l|c|c|c|}
\hline & Matching & Testing & Total \\
\hline \hline Random & 16.83 & 1.35 & 18.18 \\
\hline Alphabetical & 14.42 & 1.34 & 15.76 \\
\hline Overlap & 13.71 & 1.34 & 15.05 \\
\hline Best non-clustered & 33.28 & 6.34 & 39.62 \\
\hline
\end{tabular}

Table 3.1: Running time of the clustering algorithms on the combined query set (in seconds).

In Table 3.2, we show the number of superqueries created by each clustering algorithm, the number of postings in the resulting inverting index, and the number of accumulators created during the matching process. As we see, clustering decreases the number of index postings by about $40 \%$, but the number of accumulators created during the matches is reduced by a factor of up to 20 . This is because clustering is most effective for large query pools that have a significant number of queries with the same least common query term that can be combined.

\begin{tabular}{|l|c|c|c|}
\hline & (Super) queries & Postings & Accumulators \\
\hline \hline Random & 957366 & 6089165 & 8870966 \\
\hline Alphabetical & 948417 & 5784684 & 7670446 \\
\hline Overlap & 939779 & 5522510 & 6543883 \\
\hline \hline Best & 3069916 & 9239690 & 130691462 \\
\hline
\end{tabular}

Table 3.2: Comparison of the clustering algorithms and the best algorithm.

Next, we investigated if there are benefits in allowing 
larger superqueries with up to 64,96 , and 128 terms. We observed that there is a slight benefit in allowing up to 64 terms, but for 96 and 128 terms any further gains are lost to additional testing time. We also tried to improve the greedy approach based on overlap by looking further ahead at the next 2 and 3 sets that can be added, as opposed to a strictly one step at a time approach. However, we did not observe any measurable gains, indicating that maybe the overlap approach is already close to optimal. We ran the clustering algorithms on the different selectivity ranges, introduced in Section 2.3, which showed that again queries with many results are more expensive to match (we omit the figures due to space limitations). Finally, we summarize our results by showing the throughput rates in documents per second obtained by our various matching algorithms in Figure 3.1, on the Excite set and the combined set of Excite and AltaVista queries. We see that overall, throughput increases by more than a factor of 20 when all techniques are combined.

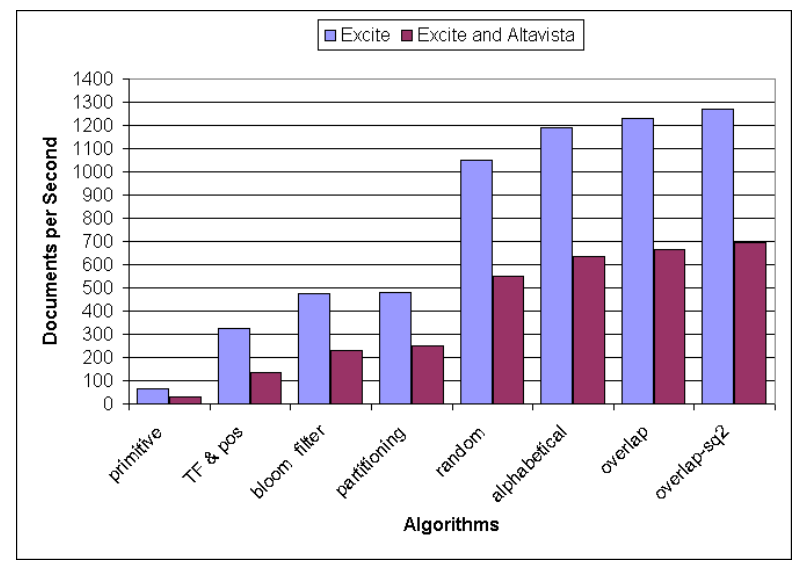

Figure 3.1: Number of documents processed per second for Excite and for the combined query set. (In overlapsq2, the superqueries can have up to 64 terms, using two unsigned integers.)

\section{Related Work}

Our work is most closely related to the SIFT project in [11], which also focuses on keyword queries and uses an inverted index structure on the queries. In SIFT, the emphasis is on ranked queries and the queries are represented in the vector space model using OR semantics. Thus, users can specify term weights and a relevance threshold (e.g., cosine measure) that are then used in identifying matching documents.

A main memory algorithm for matching events against subscriptions is proposed in [8], where an event is an attribute/value pair, and a subscription is a conjunction of (attribute, comparison operator, constant) predicates.
The proposed algorithm also employs a clustering approach. The created clusters have access predicates, where an access predicate is defined as a conjunction of equality predicates. In our approach, we create clusters with new artificial superqueries. The scenario we consider is different, as the terms in the queries as well as the content of the incoming documents are keywords.

Another body of related work is in the area of contentbased networking and publish/subscribe communication systems $[1,6]$. In this model, subscribers specify their interests by conjunctive predicates, while sources publish their messages as a set of attribute/value pairs. The goal is to efficiently identify and route messages to the interested subscribers $[5,4]$. The forwarding algorithms used by the routing nodes are related to our query processing algorithm; see [7]. Previous related work exists in the database literature about triggers and continuous queries; in stream processing and XML filtering systems.

\section{Acknowledgments}

We thank the anonymous Usenix reviewers and our shepherd John Reumann for their comments and insights.

\section{References}

[1] R. Baldoni, M. Contenti, and A. Virgillito. The evolution of publish/subscribe communication systems. In Future Directions of Distributed Computing, volume 2584 of LNCS. Springer, 2003.

[2] B. Bloom. Space/time trade-offs in hash coding with allowable errors. Communications of the ACM, 13(7):422-426, 1970.

[3] A. Broder and M. Mitzenmacher. Network applications of bloom filters: A survey. In Proc. of the 40th Annual Allerton Conf. on Communication, Control, and Computing, pages 636-646, 2002.

[4] F. Cao and J. P. Singh. Efficient event routing in content-based publish-subscribe service networks. In Proc. of IEEE Infocom Conf., 2004.

[5] A. Carzaniga, M. J. Rutherford, and A. L. Wolf. A routing scheme for content-based networking. In Proc. of IEEE Infocom Conf., 2004.

[6] A. Carzaniga and A. L. Wolf. Content-based networking: A new communication infrastructure. In NSF Workshop on an Infrastructure for Mobile and Wireless Systems, 2001.

[7] A. Carzaniga and A. L. Wolf. Forwarding in a content-based network. In Proc. of ACM Sigcomm, 2003.

[8] F. Fabret, H. A. Jacobsen, F. Llirbat, J. Pereira, K. A. Ross, and D. Shasha. Filtering algorithms and implementation for very fast publish/subscribe systems. In Proc. of ACM Sigmod Conf., 2001.

[9] J. Pereira, F. Fabret, F. Llirbat, and D. Shasha. Efficient matching for web-based publish/subscribe systems. In Conf. on Cooperative Information Systems, 2000.

[10] I. H. Witten, A. Moffat, and T. C. Bell. Managing Gigabytes: Compressing and Indexing Documents and Images. Morgan Kaufmann, second edition, 1999.

[11] T. W. Yan and H. Garcia-Molina. The SIFT information dissemination system. ACM Transactions on Database Systems, 24(4):529-565, 1999. 